\title{
Smeared Concept as a General Methodology in Finite Element Modeling of Physical Fields and Mechanical Problems in Composite Media
}

\author{
M. Kojic $\mathbf{c}^{1,2,3}$
}

${ }^{1}$ Bioengineering Research and Development Center BioIRC Kragujevac, 6 Prvoslava Stojanovica Street, 3400 Kragujevac, Serbia.

${ }^{2}$ Houston Methodist Research Institute, The Department of Nanomedicine, 6670 Bertner Ave., R7 117, Houston, TX 77030,USA.

${ }^{3}$ Serbian Academy of Sciences and Arts, 35 Knez Mihailova Street, 11000 Belgrade, Serbia. e-mail: mkojic42@gmail.com

\begin{abstract}
A generalization of the smeared concept for field problems, published in recent papers of the author and his collaborators, is presented in the paper. A composite smeared finite element CSFE is formulated. This generalization can serve as a theoretical background for further applications. A selected numerical example, related to convective-diffusive mass transport within a cancerous tissue, illustrates efficiency and accuracy of the smeared models. Further, a smeared methodology is extended to mechanical problems. A theoretical background is given in detail, with introducing a composite smeared finite element for mechanics CSFEM, which can further be tested and modified. Finally, a consistent derivation is presented for the continuum constitutive tensor corresponding to a fibrous structure.
\end{abstract}

Keywords: smeared finite element methodology, field problems, mechanical problems

\section{Introduction}

The space is, with exception of a vacuum, occupied by material substances (solid, fluid, gases) and generally contains different material constituents. There special cases in engineering or bioengineering where the space is filled with one constituent only. Then, in solving problems of physical fields or mechanical deformations we deal with one constitutive law fundamental for that constituent. By applying the basic principles and numerical methods (such as the finite element method, FEM) it is possible to obtain solutions for a given problem. However, in physics, engineering or medical science, composite media are mostly present, such as in geology (soils, rocks), according to Sowers (1979), or in living organisms (Kojic et. al 2008) (tissue, biological fluids), and the mathematical and computational models are more complex. We further consider only these models, where computational models are based on the FEM.

One approach in modeling problems within complex, composite media is to divide the entire domain into subdomains occupied by a constituent, with the corresponding laws and properties, and formulate the subdomain models. Then, the next step is to model the interactions at the domains common boundaries and generate the model for the entire system and solve for the unknowns describing the problem. This approach requires, in general, significant effort to specify 
all internal boundaries between domains, if they are visible; often, it is not possible, such as, for example, in tissue composed of complex extracellular space, cells, cell organelles, etc. It is therefore desirable to have simpler models, which take into account media complexity, but which give satisfactory accuracy.

In last few years the author introduced a smeared concept for the physical fields in biomechanics and implemented, in collaboration with his team, into the code PAK (Kojic et. al 2016a). Here are listed the basic references where this methodology has been employed (Kojic et. al 2016b, 2017a, 2017b, 2017c, 2018, Milosevic et. al 2018a, 2018b). In this paper we summarize the previous work in a way to present the methodology as general, with appropriate generalized mathematical expressions, for the field problems in composite media. Besides this, we introduce a general concept of smeared methodology for mechanics of composite media; this can serve as a background for further testing of the concept with possible modifications.

\section{Field problems - summary of the fundamental equations and FE formulation}

\subsection{Fundamental equations for the gradient driven field problems}

Diffusion. The constitutive law for diffusion is known as Fick's law,

$$
Q_{i}=-D \frac{\partial c}{\partial x_{i}}
$$

and the mass balance equation is

$$
-\frac{\partial c}{\partial t}+\frac{\partial}{\partial x_{i}}\left(D \frac{\partial c}{\partial x_{i}}\right)+q_{V}=0, \quad \text { sum on } i, i=1,2,3
$$

Here, $c$ is concentration, $Q_{i}$ flux in direction $x_{i}, D$ is diffusion coefficient and $q_{V}$ is a source term. We keep the conditions simple, without convection and assuming isotropy, for easier presentation of the smeared methodology. The generality is kept in assumption that the diffusivity $D$ can be a function of concentration, i.e. it can be $D=D(c)$.

Heat conduction. The constitutive relation is described by the Furrier law,

$$
Q_{T_{i}}=-K \frac{\partial T}{\partial x_{i}}
$$

and the balance equation is

$$
-\rho c_{T} \frac{\partial T}{\partial t}+\frac{\partial}{\partial x_{i}}\left(K \frac{\partial T}{\partial x_{i}}\right)+q_{T V}=0, \quad \text { sum on } i, i=1,2,3
$$

where $T$ is temperature, $Q_{T i}$ is thermal flux, $K(T)$ is thermal conduction coefficient, $\rho$ is density, $c_{T}$ is specific heat, and $q_{T V}$ is a source term.

Electrostatics. We start with the constitutive law

$$
J_{i}=-G \frac{\partial V_{e}}{\partial x_{i}}
$$

where $G$ is electric conductivity and $V e$ is electrical potential. The continuity equation for the current density can be derived from Maxwell Equations (1873) in a from 


$$
\frac{\partial}{\partial t}\left(\frac{\partial D_{i}}{\partial x_{i}}\right)=-\frac{\partial J_{i}}{\partial x_{i}}, \quad \text { sum on } i, i=1,2,3
$$

where the current density components $D_{i}$ can be related to the potential $V_{e}$ as

$$
D_{i}=-\varepsilon \frac{\partial V_{e}}{\partial x_{i}}
$$

where $\varepsilon$ is dielectric constant. Finally, the continuity equation is

$$
\varepsilon \frac{\partial}{\partial t} \frac{\partial^{2} V_{e}}{\partial x_{i} \partial x_{i}}=-G \frac{\partial^{2} V_{e}}{\partial x_{i} \partial x_{i}}
$$

1D-conditions. The above listed equations are applicable to the 1D-conditions, except equation (8), i.e. we have for diffusion and heat transfer,

$$
\begin{gathered}
-\frac{\partial c}{\partial t}+\frac{\partial}{\partial \bar{x}}\left(D \frac{\partial c}{\partial \bar{x}}\right)+q_{V}=0 \\
-\rho c_{T} \frac{\partial T}{\partial t}+\frac{\partial}{\partial \bar{x}}\left(K \frac{\partial T}{\partial \bar{x}}\right)+q_{V}=0
\end{gathered}
$$

where $\bar{x}$ is the axis of propagation.

Transport through membranes. Different domains of a composite media are often separated by membranes, or walls in case of the $1 \mathrm{D}$ domains. It is necessary for the presentation of the smeared methodology to list fundamental equations for transport through boundaries of the domains; in living organisms these are vessel walls or cell and organelle membranes. In case of diffusion and heat transfer these relations can be written as:

$$
Q_{w}=D_{w}\left(c_{\text {in }}-c_{\text {out }}\right)
$$

and

$$
Q_{T_{w}}=K_{w}\left(T_{\text {in }}-T_{\text {out }}\right)
$$

with the flux oriented outward (from in to out); $D_{w}$ and $K_{w}$ are wall diffusivity and conductivity, respectively. In case of electrical field, the wall electrical flux relies on the so called cable theory, according to Winslow (1992). The outlet electrical flux (current density) can be expressed as

$$
I_{m}=G_{m}\left(V_{\text {in }}-V_{\text {out }}\right)+C_{m}\left(\frac{\partial V_{\text {in }}}{\partial t}-\frac{\partial V_{\text {out }}}{\partial t}\right)
$$

where $G_{m}$ is membrane conductivity and $C_{m}$ is specific membrane (wall) capacitance.

\subsection{Finite element formulation}

In order to transform the above governing equations into the FE equations of balance for a single finite element, we implement a standard Galerkin weighting method (Kojic et. al 2008). The incremental-iterative form of balance for a time step $\Delta t$ and iteration i can be derived in the form

$$
\left(\frac{1}{\Delta t} \mathbf{M}+\mathbf{K}\right)^{(i-1)} \Delta \boldsymbol{\Phi}^{(i)}=\mathbf{Q}^{e x t}+\mathbf{Q}^{V}-\frac{1}{\Delta t} \mathbf{M}^{(i-1)}\left(\boldsymbol{\Phi}^{(i-1)}-\boldsymbol{\Phi}^{t}\right)-\mathbf{K}^{(i-1)} \boldsymbol{\Phi}^{(i-1)}
$$


where

$$
\begin{aligned}
& M_{I J}=\int_{V} c_{m} N_{I} N_{J} d V \\
& K_{I J}=\int_{V} D N_{I, k} N_{J, k} d V, \quad \text { sum on } k, k=1,2,3 \\
& Q_{I}^{V}=\int_{V} N_{I} q_{V} d V
\end{aligned}
$$

where $\boldsymbol{\Phi}$ stands for concentration, temperature or electrical potential as nodal variables; $N_{I}$ are interpolation functions, $V$ is element volume; $c_{m}$ is mass coefficient $\left(=1\right.$ for diffusion and $=\rho c_{T}$ for heat conduction); instead of $D$ we have $K$ for heat transfer and $G$ for electrical field. For electrical potential we have that the "mass" matrix is

$$
M_{I J}=\varepsilon \int_{V} N_{I, k} N_{J, k} d V, \quad \text { sum on } k, k=1,2,3
$$

Note that for 1D problems the equations have the same form as the above, with no summation on $k(k=1)$ and that the element volume is $V=A L$, where $A$ is cross-sectional area and $L$ is the element length.

For modeling transport through the membranes (walls) we introduced connectivity elements at each node (Fig. 1), by using double nodes at the same space position, with one node belonging to one side of the boundary between two domains. The connectivity element does not have the length, only cross section which is equal to the boundary surface belonging to the FE node. Then the balance equation of the form (14) is applicable. Assuming linear distribution of concentration or temperature along the membrane thickness, we have the "mass" and transport matrices as

$$
\begin{aligned}
& M_{11}=M_{22}=\frac{1}{3} c_{m m} A_{m} h_{m}, \quad M_{12}=M_{21}=\frac{1}{6} c_{m m} A_{m} h_{m} \\
& K_{11}=K_{22}=-K_{12}=-K_{21}=D_{w} A_{m}
\end{aligned}
$$

where $A_{m}$ is the area of the surface belonging to the node, $h_{m}$ is the membrane thickness (of course, in case of heat conduction we have $K_{w}$ instead $D_{w}$, and $G_{m}$ for electrical conduction); $\mathrm{c}_{\mathrm{mm}}=1$ for diffusion, and $c_{m m}=\rho_{m} c_{T m}$ for heat conduction. In case of electrical conduction the non-zero terms of the "mass" matrix are

$$
M_{11}=M_{22}=A_{m e m} C_{m}
$$

\section{Smeared model for field problems}

\subsection{A general formulation of composite smeared finite element (CSFE)}

We first describe the model which includes continuum domains, 1D domains and connectivity elements. Schematics of such FE model, which will further be called 'detailed model', is shown in Fig. 1. It is assumed that each compartment has its own FE mesh of continuum elements, while 1D domains (as blood vessels or fibers) have their own 1D finite elements with the coordinate axes depicted at one of these compartments. The connectivity element at the node common for the two domains is shown in space and enlarged (at the top of the figure), as well as the connectivity element A,B for coupling $1 \mathrm{D}$ and surrounding domain. It can be seen that the detailed model requires significant effort for the model generation, and in case of complex medium as tissue, the model generation would be an impractical or even impossible task. This 
task would be much more demanding if instead connectivity elements, continuum FEs are employed for membranes.

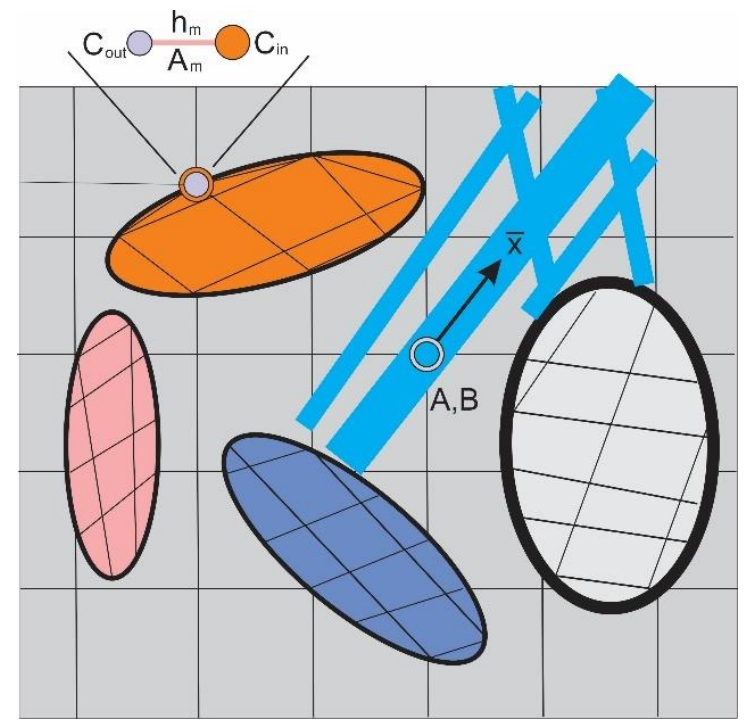

Fig. 1. Schematic of detailed model of a composite medium, 2D representation (diffusion is assumed).

The goal of smeared model is to formulate a composite continuum finite element which should include all constituents (continuum and 1D) in a way that the true physical fields, corresponding to detailed model, are represented in a smeared (a kind of average) sense, with, of course, satisfactory accuracy. A schematic of the smeared model, for the same detailed model in Fig. 1, is shown in Fig. 2a, where continuum elements are present only. There are few conceptual steps to formulate such composite smeared finite element (CSFE).

First, it is necessary to transform 1D fundamental equations into the corresponding continuum representation. The derivation of the Darcy and diffusion tensors is given in (Kojic et. al 2017a); here we present a generalized form of that derivation. We start with the fact that the gradient driven flux in a $1 \mathrm{D}$ domain (compartment) $K$, with the cross-section $A_{K}$ - due to a gradient $\partial \phi / \partial x_{i}$ - can be expressed as

$$
\bar{Q}_{K(i)}=D_{K} A_{K}\left(\frac{\partial \phi}{\partial \bar{x}}\right)_{(i)}=D_{K} A_{K} \frac{\partial \phi}{\partial x_{i}} \frac{\partial x_{i}}{\partial \bar{x}}=D_{K} A_{K} \ell_{K i} \frac{\partial \phi}{\partial x_{i}}, \quad \text { no sum on } i
$$

where $D_{K}$ is the transport coefficient for the domain, $\partial \phi / \partial \bar{x}$ is the gradient of the field $\phi$ in the direction $\bar{x}$ of the 1D domain, and $\ell_{K i}$ are directional cosines of the $\bar{x}$ axis. Further, the component $Q_{K_{j}}$ in direction $x_{j}$ due to all gradients of the $\partial \phi / \partial x_{i}$ is

$$
Q_{K j}=\ell_{K j} \sum_{i} \bar{Q}_{K(i)}=D_{K} A_{K}^{\ell} K_{j}^{\ell} K i \frac{\partial \phi}{\partial x_{i}}, \quad \text { sum on } i, i=1,2,3
$$

and the flux per unit area due to all $1 \mathrm{D}$ domains is 


$$
q_{j}=\frac{1}{A_{t o t}} \sum_{K} Q_{K j}=\frac{1}{A_{t o t}} \sum_{K} D_{K} A_{K} \ell_{K i} \frac{\partial \phi}{\partial x_{i}} \ell_{K j}, \quad \text { sum on } i, i=1,2,3
$$

where

$$
A_{\text {tot }}=\sum_{K} A_{K}
$$

is the total area of all 1D compartments. Therefore, the transport tensor corresponding the 1D domains is

$$
D_{i j}=\frac{1}{A_{t o t}} \sum_{K} D_{K} A_{K} \ell_{K i} \ell_{K j}
$$

The next important statement in the CSFE formulation is that the volume of each domain has its own field within the corresponding domain volume. Hence, the FE node of the CSFE has number of nodal variables $\phi^{K}$ ('degrees of freedom') equal to the number of domains $N_{d}$, as shown in Fig. 2b. The domain volume $V_{K}$ is related to the total space volume as

$$
V_{K}=r_{V}^{K} V, \text { and } \mathrm{d} V_{K}=r_{V}^{K} d V
$$

where $r_{V}^{K}$ is the volumetric fraction. Finally, we include connectivity elements to couple corresponding domains. Namely, at each node are specified connectivity elements coupling two domains, according to the above described connectivity elements. The cross-sectional area $A_{J K}$ of a connectivity element at node $J$ can be expressed in the form
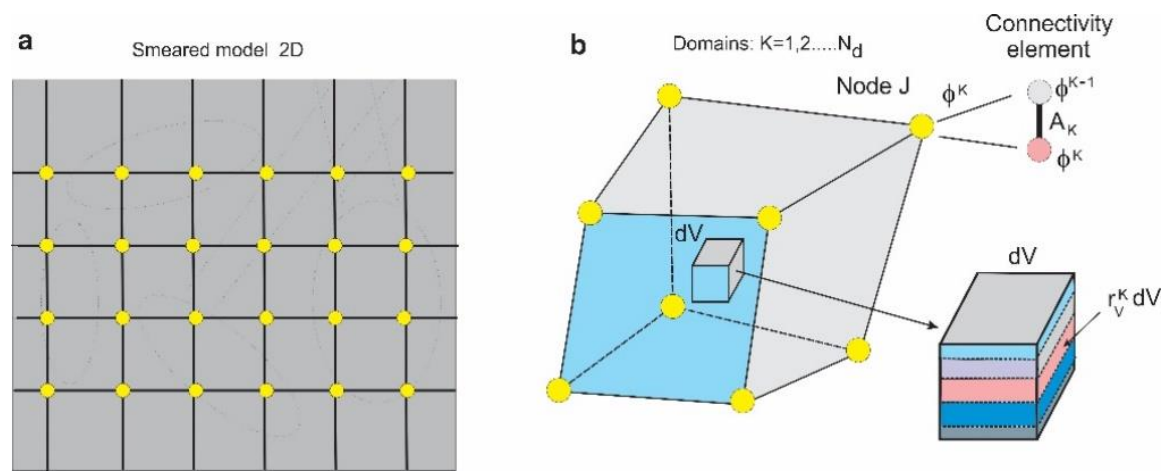

Fig. 2. Schematic of the smeared model. a) Smeared FE mesh for the detailed model in Fig. 1;

b) Composite smeared finite element CSFE with different domains and nodal 'degrees of freedom' $\phi^{K}$, and connectivity element at node $J$ between two domains.

$$
A_{J K}=\left(r_{A V}^{K} V_{K}\right)_{J}=\left(r_{A V}^{K} r^{K} V_{K}\right)_{J}
$$

where $r_{A V}^{K}$ is the area coefficient, i.e.

Next, we write the incremental equation (21) in the form

$$
r_{A V}^{K}=\frac{A_{K}}{V_{K}}
$$


and $\left(V_{K}\right)_{J}$ is the volume belonging to the node $J$. Note that all the surfaces, volumes and the volumetric and area ratios, are assigned to nodes, which in practical application is convenient to model any non-homogenous properties.

The above concept has been implemented to diffusion (including convection) and fluid transport through capillary network and tissue, with demonstration of accuracy of the smeared methodology (Kojic et al. 2017a, 2017b, 2018). To improve the smeared model accuracy, a correction function was introduced in (Milosevic et al. 2018a). Additional effects present in drug delivery, such as partitioning, can be included in the connectivity elements, as shown in our references. The smeared model can be extended to composite media with fibers in a way shown in (Milosevic et al. 2018b).

Here we demonstrate accuracy of the smeared model on one example. Other applications are given in the cited references.

\subsection{A Numerical example - a tissue sample from a pancreatic tumor}

We have selected a small 50x50 micron size 2D tissue sample taken from images of cancerous tissue (E. J. Koay, MD Anderson Cancer Center, Houston, Kojic et al. 2018) shown in Fig. 3a. It was assumed that there are two types of cells, with different cytosol properties, and with three organelles within each cell. First group is assumed to be healthy cells ( 24 cells), second group is assumed to be tumor cells ( 21 cells). Contours of cells and organelles are shown in Fig. 3b. It is assumed that there are 6 capillaries (blue contours), 4 lymph nodes (red contours), and 45 cells inside the model. Model geometrical data are as follows (lengths in microns):

Capillaries: 6 , Diameter $d_{\text {mean }}=3.97$, wall thickness $=0.62$, vol fraction $r_{V}=0.0247$

Tissue: 50x50 $\quad \mathrm{A}_{\text {total }}=2500, \quad \mathrm{~A}_{\text {tissue }}=(1-0.0247) * \mathrm{~A}_{\text {total }}=2438$

Cell 1 (24): $r_{V}=0.307, D_{\text {mean }}=6.30$

Organelles: 1) $\left.\left.\mathrm{r}_{\mathrm{V}}=0.334, \mathrm{D}_{\text {mean }}=3.64 ; 2\right) \mathrm{r}_{\mathrm{V}}=0.034, \mathrm{D}_{\text {mean }}=1.16 ; 3\right) \mathrm{r}_{\mathrm{V}}=0.03, \mathrm{D}_{\text {mean }}=1.10$

Cell 2 (21): $r_{V}=0.257, D_{\text {mean }}=6.16$

Organelles: 1) $\left.\left.\mathrm{r}_{\mathrm{V}}=0.257, \mathrm{D}_{\text {mean }}=3.17 ; 2\right) \mathrm{r}_{\mathrm{V}}=0.051, \mathrm{D}_{\text {mean }}=1.39 ; 3\right) \mathrm{r}_{\mathrm{V}}=0.056, \mathrm{D}_{\text {mean }}=1.46$

a

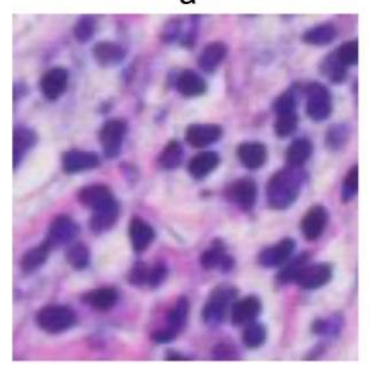

b

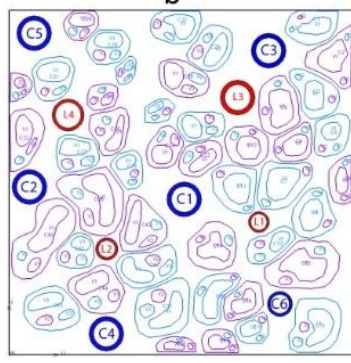

C

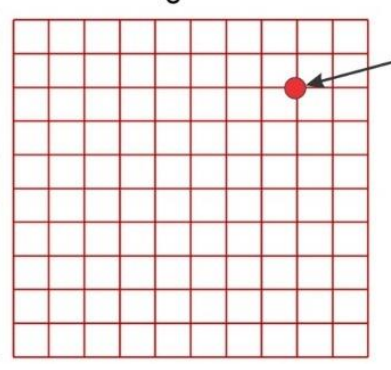

DOF Concs

Ex cell Cyt-Cell 1

Org 1
Org 2

Org 3

Cyt-Cell 2

Org 1

Org 2 Total 9

Fig. 3. Diffusion 2D model of tissue a) Image of pancreatic cancer tissue, b) Contours of capillaries, lymph, cells and organelles; c) Smeared model with nodal concentrations to be calculated.

Detailed model: Number of FE nodes: 69457, FE elements in Cells: 41763, FE nodes in cells $\sim 42000$, FE elements in capillary domain: 4290, FE nodes $\sim 4400$, FE elements in extracellular space (tissue) domain: 18486. Number of equations in detailed (true) model $\sim 87943=18486+$ 69457 (with pressures in ECM and concentration in cells) 
Number of equations in smeared model $\sim 1200$

Diffusion coefficients and partitioning within cells:

- Cell1: $\mathrm{D}_{\mathrm{C} 1}=\mathrm{D}_{\mathrm{v} 1}=\mathrm{D}_{\mathrm{v} 2}=\mathrm{D}_{\mathrm{v} 3}=\mathbf{1 0 0}, \mathrm{P}=\mathbf{1}$

- Cell2: $\mathrm{D}_{\mathrm{C} 2}=\mathrm{D}_{\mathrm{v} 1}=\mathrm{D}_{\mathrm{v} 2}=\mathrm{D}_{\mathrm{v} 3}=\mathbf{1 0 0}, \quad \mathrm{P}=\mathbf{1 0}$ (at both extracellular/cell and intercellular/organelle border)

Pressure field of detailed and tissue domain of smeared model in shown in Fig. 4. Mean pressure in extracellular space of the detailed model is $0.57 \mathrm{~Pa}$, while it is $0.608 \mathrm{~Pa}$ when using the smeared model.

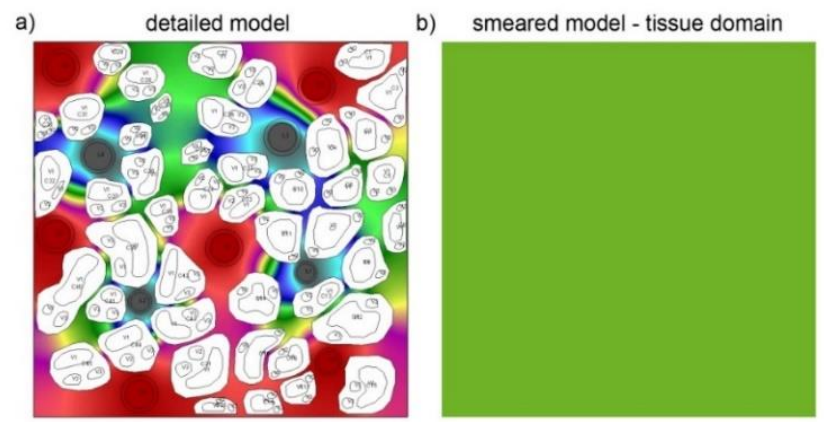

b) smeared model - tissue domain

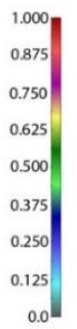

Fig. 4. Pressure field after $t=0.5 \mathrm{~s}$. a) Detailed model; b) extracellular space of smeared model.

Concentration field of detailed and tissue domain of smeared model, for two time moments $(\mathrm{t}=0.5 \mathrm{~s}$ and $\mathrm{t}=10 \mathrm{~s})$ is shown in Fig. 5 .

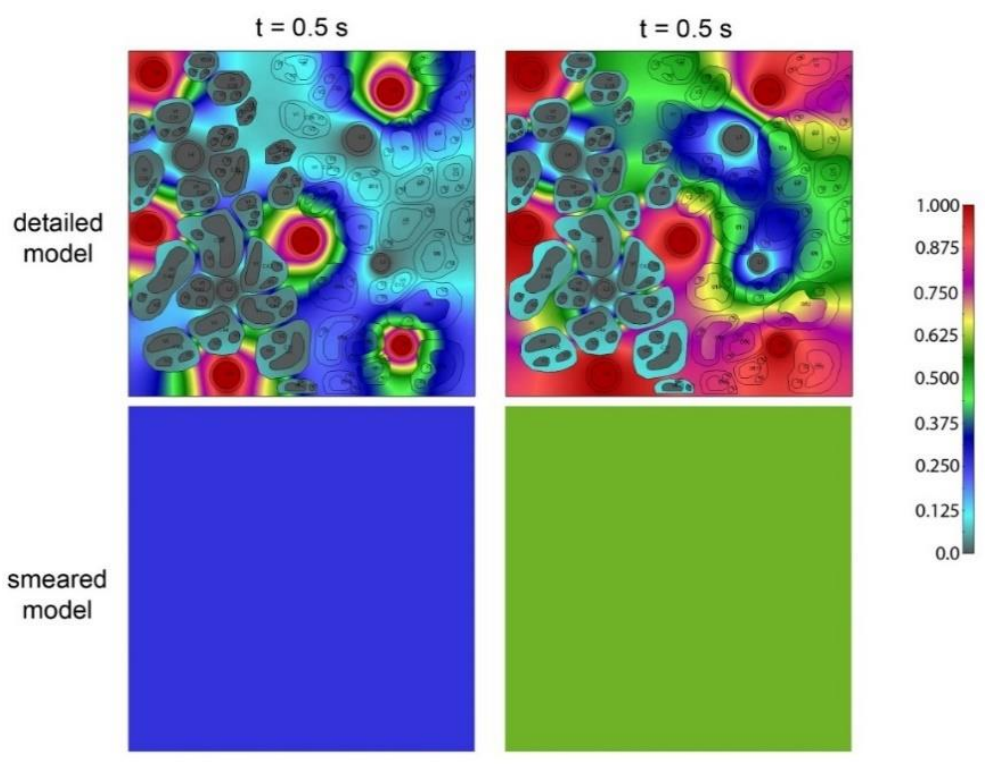

Fig. 5. Concentration field in extracellular space (tissue domain) of detailed and smeared model, for $\mathrm{t}=0.5 \mathrm{~s}$ (upper panel) and $0 \mathrm{~s}$ (lower panel). 
Accuracy of the smeared model is verified using constant and bolus function of concentration in capillaries. Results are presented in Figs. 6 and 7.
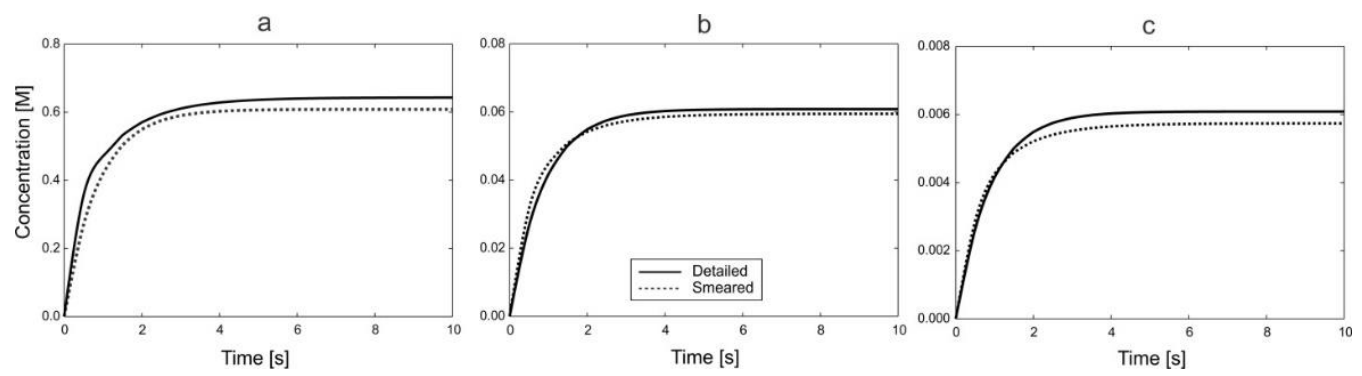

Fig. 6. Concentration vs Time for detailed and smeared model, with convection included and const $\mathrm{c}(\mathrm{t})$. a) Extracellular space and Cell type 1; b) Cell type 2 (partitioning $\mathrm{P}=10$ ); c)

Organelle 1, partitioning $\mathrm{P}=10$ of Cell type 2 .

a

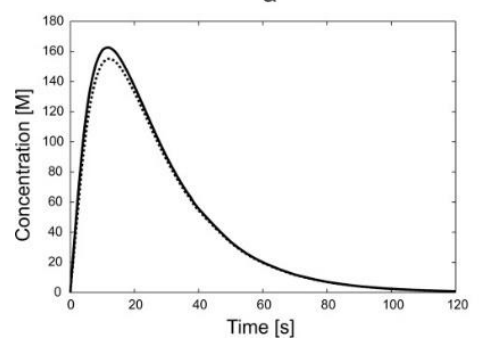

b

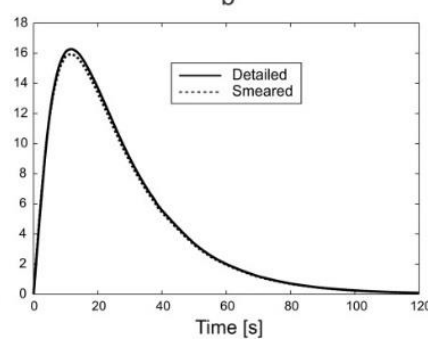

C

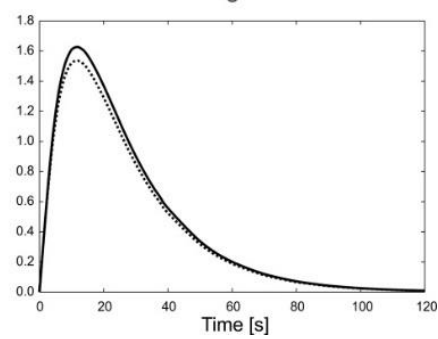

Fig. 7. Concentration vs Time for detailed and smeared model, with convection included and bolus $\mathrm{c}(\mathrm{t})\left(\mathrm{c}_{\max }\right.$ in bolus is $\left.270 \mathrm{M}\right)$ : a) Extracellular space and Cell type 1; b) Cell type 2 (partitioning $\mathrm{P}=10$ ); c) Organelle 1 of Cell type 2 (partitioning $\mathrm{P}=10$ ).

This example illustrates applicability of the smeared model, its efficiency and accuracy.

\section{Smeared method in mechanics}

In this section is presented a theoretical background for a smeared model in mechanics by considering a composite medium. The presented concept may serve as a basis for modeling tumor growth and in other applications. As a background with no work-out examples, the concept requires further testing, and refining or correction.

\subsection{Motivation}

As a motivation, consider a tissue medium composed of extracellular matrix and cells as solids, and fluid (interstitial fluid) present in the extracellular space. The cell is also a complex structure, with cell membrane surrounding the interior containing fluid component (cytosol) with the cytoskeleton as the supporting structure. The tissue medium is schematically shown in Fig. 8. As depicted in the figure, the stresses presented in this mechanical system consist of:

- $\quad$ stress in the extracellular space $\boldsymbol{\sigma}^{e}$, which can be expressed as $\boldsymbol{\sigma}^{e}=\boldsymbol{\sigma}^{e}+\boldsymbol{\sigma}_{m}^{e}$ where $\boldsymbol{\sigma}^{\prime e}$ is deviatoric stress and $\boldsymbol{\sigma}_{m}^{e}$ is the mean stress; 
- $\quad$ stress in fluid $\boldsymbol{\sigma}^{f}=\boldsymbol{\sigma}_{\mu}^{f}-\mathbf{p}^{f}$, where $\boldsymbol{\sigma}_{\mu}^{f}$ is viscous stress and $\mathbf{p}^{f}$ is fluid pressure;

- $\quad$ stress in cells $\boldsymbol{\sigma}^{c}$ which also can be decomposed into deviatoric $\boldsymbol{\sigma}^{c}$ and pressure $\mathbf{p}^{c}$ (negative mean stress) $\boldsymbol{\sigma}^{c}=\boldsymbol{\sigma}^{c}-\mathbf{p}^{c}$;

- $\quad$ sliding stresses: between fluid and extracellular matrix $\tau^{e f}$, between cells and fluid $\tau^{c f}$ , and between cells and extracellular matrix $\tau^{c e}$.

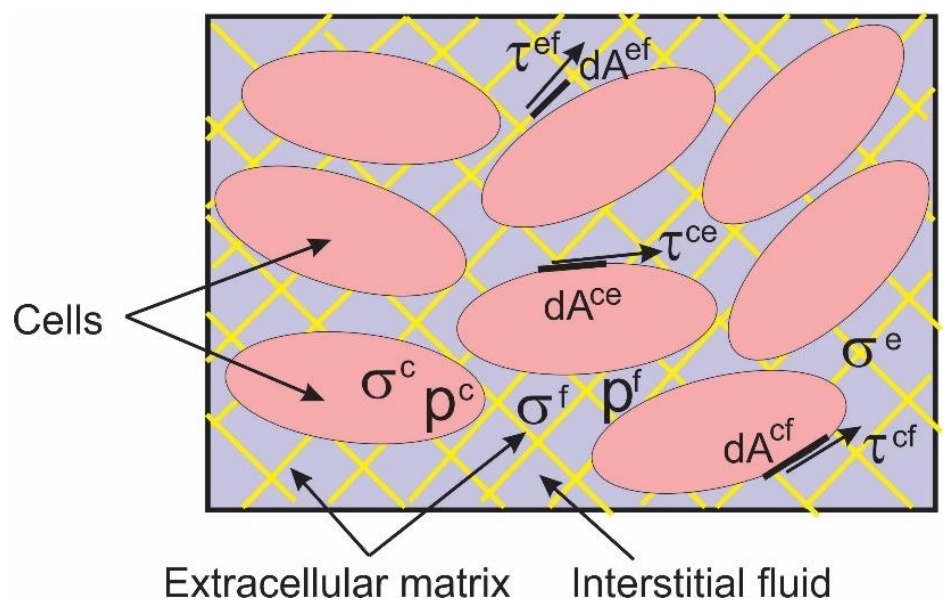

Fig. 8. Schematic of tissue composed of extracellular space with: extracellular matrix and interstitial fluid, and cells.

In resisting to the external loading, all these stresses will produce a mechanical work. So, virtual power, corresponding to the virtual velocities in the entire system; and within elementary volumes of constituents, and at elementary sliding surfaces, is:

$$
\begin{aligned}
\delta W & =\sigma_{i j}^{e} \delta \dot{e}_{i j}^{e} d V^{e}+\sigma_{i j}^{f} \delta \dot{e}_{i j}^{f} d V^{f}+\sigma_{i j}^{c} \delta \dot{e}_{i j}^{c} d V^{c} \\
& +\tau^{e f} \delta v_{r e l}^{e f} d A^{e f}+\tau^{c f} \delta v_{r e l}^{c f} d A^{c f}+\tau^{c e} \delta v_{r e l}^{c e} d A^{c e}
\end{aligned}
$$

where $\dot{e}_{i j}^{e}, \dot{e}_{i j}^{f}$ and $\dot{e}_{i j}^{c}$ are strain rates within the constituents; $v_{r e l}^{e f}, v_{r e l}^{c f}$ and $v_{r e l}^{c e}$ are relative velocities at the contact surfaces; $d V^{e}, d V^{f}$ and $d V^{c}$ are elementary volumes; and $d A^{e f}, d A^{c f}$ and $d A^{c e}$ are elementary contact surfaces. This expression represents a fundamental relation for the development of computational scheme. We will further introduce a generalization of the above model and define some details for the formulation of a smeared concept in mechanics of composite media.

\subsection{A general formulation of the expression for virtual power for composite media}

Here are introduced several assumptions for the formulation of a general smeared model:

a) There is a supporting medium (or the basic medium, with the index ' $b$ ') in which are embedded other deformable domains. In Fig. 9 these domains are denoted as domains 1,2 $N_{d}$. 
b) The velocity within a domain ' $k$ ' is $\mathbf{v}^{k}$. At the boundary between a domain and the supporting medium, velocities for the supporting medium and for the domain $k$ are expressed in the local coordinate system,

$$
\mathbf{v}^{b}=v_{n}^{b} \mathbf{n}^{k}+v_{t}^{b} \mathbf{t}^{k}, \quad \mathbf{v}^{k}=v_{n}^{k} \mathbf{n}^{k}+v_{t}^{k} \mathbf{t}^{k}
$$

where $v_{n}^{b}, v_{n}^{k}$ and $v_{t}^{b}, v_{t}^{k}$ are velocity components in direction of the boundary normal $\mathbf{n}^{k}$ and tangent $\mathbf{t}^{k}$ to the domain. We impose the condition that normal components are the same, while there is a relative velocity $v_{t}^{k b}$, i.e.

$$
v_{n}^{b}=v_{n}^{k}, \quad v_{t}^{b k}=v_{t}^{k}-v_{t}^{b}
$$

a) At the boundary between two domains, we also use local coordinate system and express velocities as

$$
\mathbf{v}^{k}=v_{n}^{k} \mathbf{n}^{k}+v_{t}^{k} \mathbf{t}^{k}, \quad \mathbf{v}^{k+1}=v_{n}^{k+1} \mathbf{n}^{k}+v_{t}^{k+1} \mathbf{t}^{k}
$$

and

$$
v_{n}^{k+1}=v_{n}^{k}, \quad v_{t}^{k, k+1}=v_{t}^{k+1}-v_{t}^{k}
$$

where $v_{t}^{k, k+1}$ is relative sliding velocity of the domain ' $k+1$ ' with respect to domain ' $k$ '.

b) The stress due to sliding between a compartment ' $k$ ' and the supporting medium is $\tau^{b k}$, while between ' $k$ ' and ' $k+1$ ' is $\tau^{k, k+1}$.

c) Elementary surfaces between supporting medium and the medium ' $k$ ', and between two media are $d A^{b k}$ and $d A^{k, k+1}$, respectively.

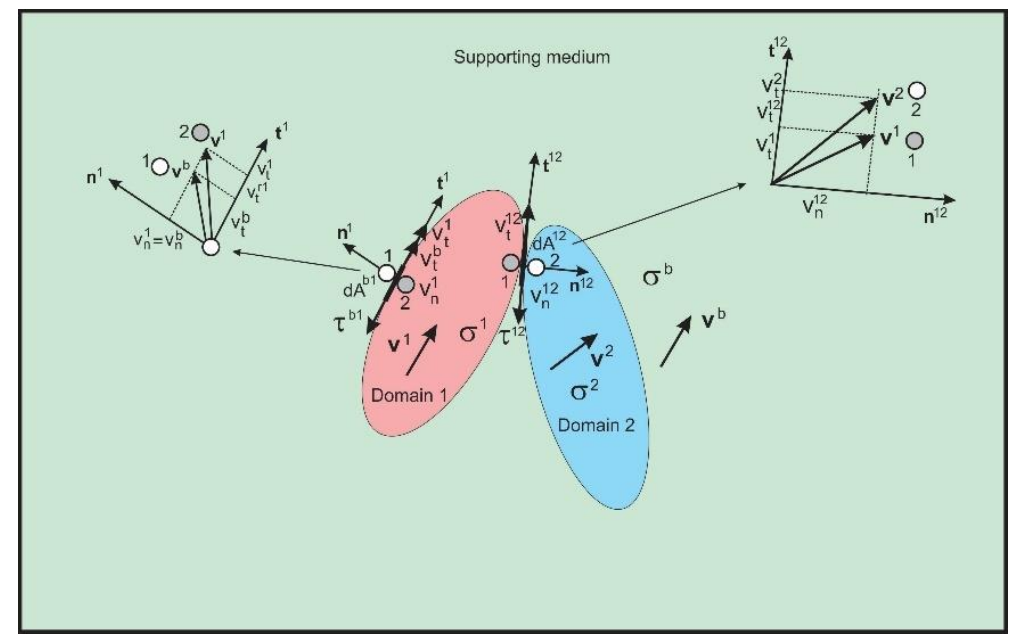

Fig. 9. Schematic of velocities and stresses at the interfaces.

The virtual power due to stresses, analogous to the expression (28), is 
$\delta W^{\text {stress }}=\sigma_{i j}^{b} \delta \dot{e}^{b} d V^{b}+\sum_{k=1}^{N_{d}} \sigma_{i j}^{k} \delta \dot{e}_{i j}^{b} d V^{k}+\sum_{k=1}^{N_{d}} \tau^{b k} \delta v_{t}^{b k} d A^{b k}+\sum_{k=1}^{N_{d}-1} \tau^{k, k+1} \delta v_{t}^{k, k+1} d A^{k, k+1}$

Virtual power due to inertial forces is

$$
\delta W^{i n}=-\rho^{b} \dot{v}_{i}^{b} \delta v_{i}^{b} d V^{b}-\sum_{k=1}^{N_{d}} \rho^{k} \dot{v}_{i}^{k} \delta v_{i}^{k} d V^{k}
$$

\subsection{Finite element formulation}

Finite element balance equations for continuum space, for a time step and iteration i, have a standard form (Kojic et. al 2008),

$$
\left(\frac{1}{\Delta t} \mathbf{M}+\mathbf{K}^{(i-1)}\right) \Delta \mathbf{V}^{(i)}=\mathbf{F}^{e x t}-\mathbf{F}^{\operatorname{int}(i-1)}+\mathbf{F}^{\text {in }(i-1)}
$$

where $\mathbf{V}$ are nodal velocities, $\mathbf{F}^{\text {ext }}$ are external forces at the element nodes; $\mathbf{F}^{\text {int }}$ and $\mathbf{F}^{\text {in }}$ are internal forces due to stresses and inertial forces. In general, the matrices and nodal vectors can be expressed as

$$
\begin{aligned}
& \mathbf{M}=\int_{V} \rho \mathbf{N}^{T} \mathbf{N} d V, \quad \mathbf{K}=\int_{V} \mathbf{B}^{T} \mathbf{C} \mathbf{B} d V \\
& \mathbf{F}^{\mathrm{int}(i-1)}=\int_{V} \mathbf{B}^{T} \boldsymbol{\sigma}^{(i-1)} d V, \quad \mathbf{F}^{\mathrm{in}(i-1)}=-\mathbf{M} \dot{\mathbf{V}}^{(i-1)}
\end{aligned}
$$

where $\mathbf{N}$ is the interpolation matrix, and $\mathbf{B}$ is the geometric matrix with the derivatives with respect to spatial coordinates; and $\mathbf{C}$ is the constitutive matrix; details are given elsewhere e.g. (Kojic et. al 2008). At the contact nodes we transform the velocities from the local to global coordinate system and include into the balance equation. For a better convergence, a transformation of the local stiffness matrix to the global system may be performed. The interaction forces, due to relative velocities at the contact boundary $k, k+1$, at a node $J$ can be modeled by introducing a connectivity element at the node, for which the balance equation is

$$
K_{I J}^{k, k+1} \Delta\left(V^{k, k+1}\right)_{J}^{t(i)}=-K_{I J}^{k, k+1}\left(V^{k, k+1}\right)_{J}^{t(i-1)}
$$

where $\left(V^{k, k+1}\right)_{J}^{t}$ is tangential velocity at node $J$ for domains $k+1$ and $k$, and the matrix $K_{I J}^{k, k+1}$ is

$$
K_{I J}^{k, k+1}=A_{J}^{k, k+1} \eta^{k, k+1}
$$

Here, $A_{J}^{k, k+1}$ is the area of the contact surface belonging to the common node $J$, and $\eta^{k, k+1}$ is the resistance (viscous) coefficient. The balance equation for connectivity elements between a domain $k$ and supporting medium has the form (36).

The condition that normal velocities at the common boundary are the same can be achieved by assigning the same equation number for both domains, as is assumed above. But, also, this can be achieved by introducing connectivity elements in normal directions (as for the tangent above), so that we introduce the balance equation of the form (36),

$$
K_{I J}^{(k, k+1)_{n}} \Delta\left(V^{k, k+1}\right)_{J}^{n(i)}=-K_{I J}^{(k, k+1)_{n}}\left(V^{k, k+1}\right)_{J}^{n(i-1)}
$$

where 


$$
K_{I J}^{(k, k+1)_{n}}=A_{J}^{k, k+1} E_{n}^{k, k+1}
$$

The elastic modulus $E_{n}^{k, k+1}$ can be taken sufficiently large to provide the desired condition in the normal directions.

\subsection{A smeared finite element formulation}

Here, we introduce a smeared FE formulation for mechanics in a way analogous to field problems described above and in our listed references, specifically in reference (Kojic et al. 2018). The concept of the smeared finite element is shown in Fig. 10.

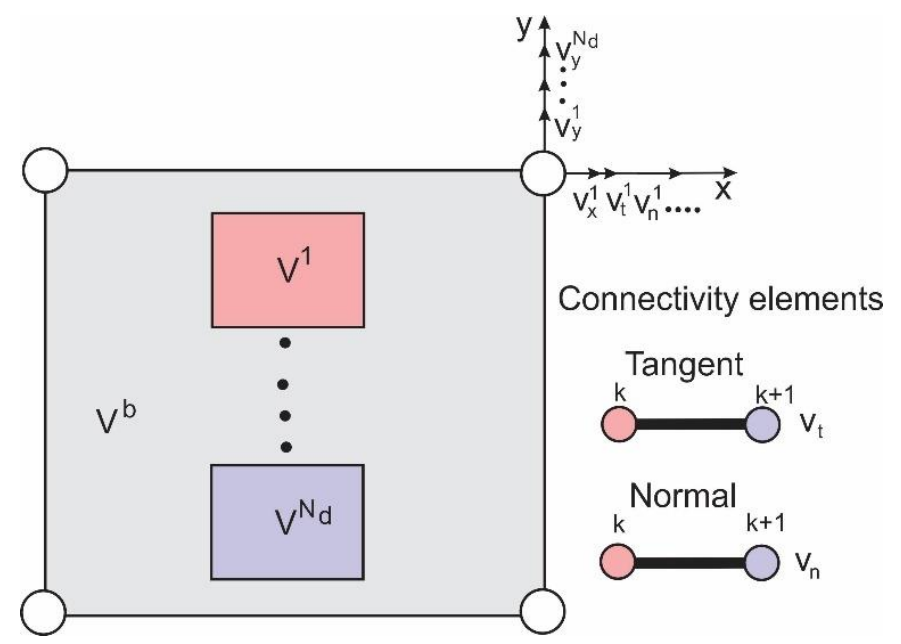

Fig 10. Composite smeared finite element for mechanics, CSFEM (2D representation). The element volume is composed of the supporting medium of volume $V^{b}$ and different domains

with volumes $V^{l}$ to $V^{N_{d}}$. The nodal degrees of freedom include velocities in coordinate directions, and tangential and normal components for the domains boundaries. Connectivity elements couple velocities at the boundaries.

The volumes of the domains are specified by the volumetric fractions as in case of field problems, so that the volumetric fraction of the supporting medium is

$$
r_{V}^{b}=1-\sum_{k=1}^{N_{d}} r_{v}^{k}
$$

The continuum balance equations for each of the domains have the form presented above for detailed model, within the domain volume $V^{k}=r_{V}^{k} V$.

The nodal tangent and normal forces, $F_{J_{i}}^{t}$ and $F_{J_{i}}^{n}$, due to connectivity elements, in directions of the global axes $x_{i}$, are

$$
F_{J i}^{t}=-K_{I J}^{k, k+1}\left(V^{k, k+1}\right)_{J}^{t(i-1)}\left(r_{A i}^{t}\right)_{J}
$$

and 


$$
F_{J i}^{n}=-K_{I J}^{(k, k+1)_{n}}\left(V^{k, k+1}\right)_{J}^{n(i-1)}\left(r_{A i}^{n}\right)_{J}
$$

where $\left(r_{A i}^{t}\right)_{J}$ and $\left(r_{A i}^{n}\right)_{J}$ are fractions of the boundary surfaces (area fractions) $A^{t}$ and $A^{n}$, in directions of the global coordinate axes $x_{i}$. In case of 2D circular domain, the area fractions are $r_{A i}^{t}=r_{A i}^{n}=2 / \pi$; in case of a rectangular domain with dimensions $a$ and $b$, with the side with dimension a lying along x-direction, the area fractions are: $r_{A x}^{t}=r_{A y}^{n}=a /(a+b)$, $r_{A y}^{t}=r_{A x}^{n}=b /(a+b)$. Note that the local stiffness matrices may be transformed into the global system for better convergence (details not given here).

Finally, we present relations which can be used for transforming 1D balance equation of supporting fibers into a continuum form. There are various media with fibrous structure, as extracellular space or cell interior with cytoskeleton (shown in Fig 11a), and, from the practical implementation, it would be desirable to have a continuum representation of a complex network of fibrous structure. A sketch of a fibrous structure is shown in Fig. 11b.

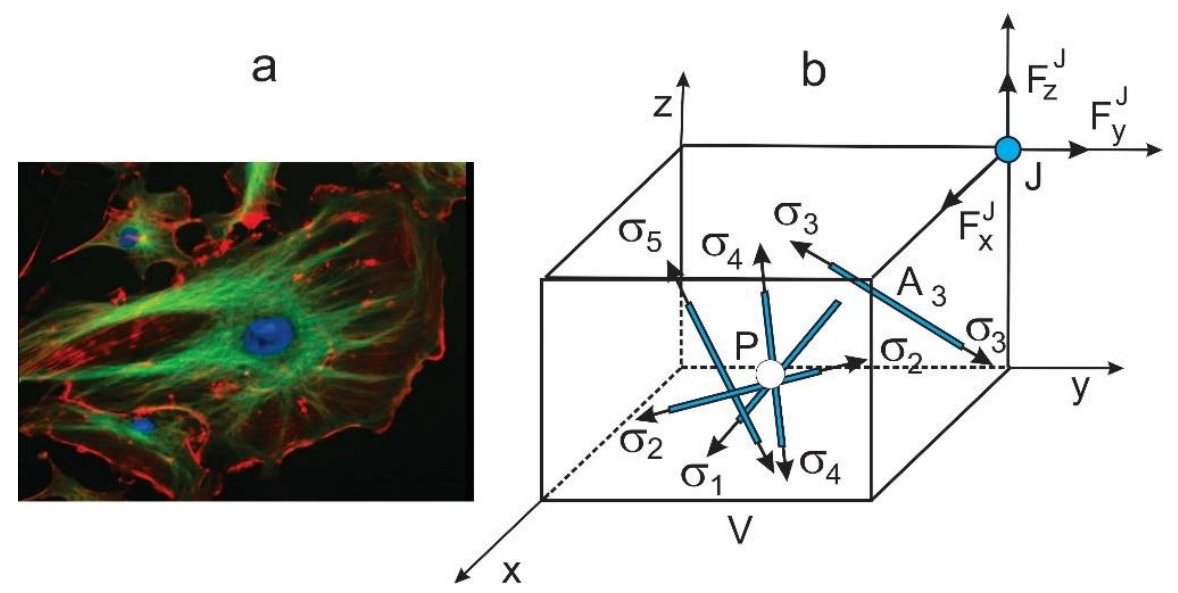

Fig. 11. Fibrous structure. a) Cytoskeleton within a cell (from Wikipedia); b) Schematic of fibrous structure within a finite element, stresses within fibers and the smeared nodal forces components equivalent to the forces within fibers.

The equivalent (smeared) nodal forces within a finite element may be derived as follows. Let strains at a Gauss point be (engineering strains - one index notation (Kojic et al. 1998, 2008), then, for a fiber $K$, the strain $e_{K}$ is

$$
e_{K}=T_{1 j}^{\varepsilon K} e_{j}
$$

where the transformation matrix $T_{1 j}^{\varepsilon K}$ contains terms with directional cosines of the fiber $K$. The stress $\sigma_{K}$ within the fiber is

$$
\sigma_{K}=E_{K} T_{1 j}^{\varepsilon K} e_{j}
$$

where $E_{K}$ is elastic modulus of the fiber (also, a nonlinear constitutive law can be used). Now, we introduce a fiber geometric coefficient $g_{K}$ as 


$$
g_{K}=\frac{1}{V_{r e f}} A_{K} L_{K}
$$

where $L_{K}$ are fiber lengths within a reference volume $V_{\text {ref }}$ (a cube with equal lengths). Stresses in the global system $\sigma_{i}^{K}$ are

$$
\sigma_{i}^{K}=T_{i 1}^{\varepsilon K} \sigma^{K}=E_{K} T_{i 1}^{\varepsilon K} T_{1 j}^{\varepsilon K} e_{j}
$$

Virtual power per volume $d V$ of the finite element (for a fiber $K$ ) is

$$
\delta W_{K}=\sigma_{i}^{K} \delta e_{i} d V_{K}=g_{K} E_{K} T_{i 1}^{\varepsilon K} T_{1 j}^{\varepsilon K} e_{j} \delta e_{i} d V=E_{i j}^{K} e_{j} \delta e_{i} d V
$$

and

$$
E_{i j}^{f i b}=\sum_{K} g_{K} E_{K} T_{i 1}^{\varepsilon K} T_{1 j}^{\varepsilon K}
$$

is the elastic constitutive tensor for all fibers. The nodal forces due to stresses within fibers are shown for a node $J$.

\section{Conclusions}

The presented generalization of the smeared concept, initiated by the author and tested in papers published recently together with his collaborators, offers a broad theoretical basis for applications in problems of physical fields. The material laws in these problems rely on the gradient governed changes in the physical space. Besides numerous examples in the cited reference which illustrated efficiency, accuracy and applicability of the smeared methodology, one typical numerical example is included in the paper.

The smeared methodology extended to mechanical problems can serve as a starting approach (with possible improvements and corrections) in further applications of this concept to various complex problems in biomedical and general engineering and science.

Acknowledgements This paper is supported by the SILICOFCM project that has received funding from the European Union's Horizon 2020 research and innovation programme under grant agreement No. 777204. This research was also funded by Ministry of Education, Science and Technological Develoipment of Serbia, grants OI 174028, III 41007 and III 45019. It was supported by the Houston Methodist Research Institute and the City of Kragujevac, Serbia.

The author acknowledges support from collaborators from the Bioengineering Research and Development Center BioIRC (particularly Dr. M. Milosevic, and also V. Simic and B. Milicevic), and from Houston Methodist Research Institute (Dr. M. Ferrari and Dr. A. Ziemys and others).

\section{References}

Kojic M, Slavkovic R, Zivkovic M, Grujovic N (1998). Finite Element Method I - Linear Analysis, Faculty of Mechanical Engineering, Kragujevac.

Kojic M, Filipovic N, Stojanovic B, Kojic N (2008). Computer Modelling in Bioengineering Theory, Examples and Software, J. Wiley and Sons. 
Kojic M, Slavkovic R, Zivkovic M, Grujovic N, Filipovic N, Milosevic M, Isailovic V (2016a). PAK-FE program for structural analysis, fluid mechanics, coupled problems and biomechanics, Bioengineering R\&D Center for Bioengineering, Faculty of Technical Science, Kragujevac, Serbia. http://www.bioirc.ac.rs/index.php/software, (accessed on 21 November 2016).

Kojic M (2016b). A review of models in bioengineering developed by group lead by Miloš Kojić in period 2006-2016, J. Serb. Soc. Comp. Mech., Vol. 10 (1), 5-19.

Kojic M, Milosevic M, Simic V, Koay EJ, Fleming JB, Nizzero S, Kojic N, Ziemys A, Ferrari M (2017a). A composite smeared finite element for mass transport in capillary systems and biological tissue, Comp. Meth. Appl. Mech. Engrg., 324, 413-437, https://doi.org/10.1016/j.cma.2017.06.019.

Kojic M, Milosevic M, Simic V, Stojanovic D, Uskokovic P (2017b). A radial 1 D Finite Element for Drug Release from Drug Loaded Nanofibers, J. Serb. Soc. Comp. Mech., Vol 11, No.1, pp. 82-93.

Kojic M, Milosevic M, Simic V, Koay EJ, Kojic N, Ziemys A, Ferrari M (2017c). Extension of the Composite Smeared Finite Element (CSFE) to Include Lymphatic System in Modeling Mass Transport in Capillary Systems and Biological Tissue, J. Serb. Soc. Comp. Mech., Vol 11, No.2, pp. 108-120.

Kojic M, Milosevic M, Simic V, Koay EJ, Kojic N, Ziemys A, Ferrari M (2018). Multiscale smeared finite element model for mass transport in biological tissue: from blood vessels to cells and cellular organelles, Computers in Biology and Medicine, Vol 99, DOI: 10.1016/j.compbiomed.2018.05.022, pp .7-23.

Maxwell JC (1873). A treatise on electricity and magnetism, Clarendon Press.

Milosevic M, Simic V, Milicevic B, Koay EJ, Ferrari M, Ziemys A, Kojic M (2018a). Correction function for accuracy improvement of the Composite Smeared Finite Element for diffusive transport in biological tissue systems, Comp. Meth. Appl. Mech. Engrg., Vol 338, August 2018, doi.org/10.1016/j.cma.2018.04.012, pp 97-116.

Milosevic M, Stojanovic D, Simic V, Milicevic B, Radisavljevic A, Uskokovic P, Kojic M (2018b). A Computational Model for Drug Release from PLGA Implant, Materials, Vol. 11, November 2018, https://doi.org/10.3390/ma11122416, pp 1-17.

Sowers GF (1979). Introductory Soil Mechanics and Foundations: Geotechnical Engineering, Prentice-Hall.

Winslow Raimond L (1992). Theoretical Foundations of Neural Modeling, BME 580.681, Depts Biomed. Engrg and Computer Science, The Johns Hopkins Univ., Sch. Medicine and Whiting Sch. Engrg. 